life, planning and communication for advanced cancer patients. Little is known about the impact of PCI on hospital admissions.

$800+$ consultations per year occur in Combined Oncology and Palliative Care clinics (COPC) at Royal Cornwall Hospital, each patient seen by the appropriate specialty consultant. Less COPC patients received radical or potentially curative treatment. This study examines the influence of COPC versus Standard Oncology (SO) clinics on hospital admissions.

Methods Fifty-five COPC patients with Breast, Prostate or Gastro-intestinal cancers were compared with fifty-eight attending SO (matched diagnoses). Number of non-elective hospital admissions and length of stay (LOS) were examined during a 12 month period.

Results In the COPC there were 45 admissions (LOS 4.3 days), and 70 admissions in the SO (LOS 5.6 days). The main reason for admission was a symptom/complication of diagnosis: COPC $84 \%$; SO $86 \%$. Of these complications of treatment were the reason in $10.5 \%$ of COPC admissions; but in $26.7 \%$ of SO admissions. $87 \%$ of COPC patients, and $81 \%$ of SO patients were discharged home.

45\% COPC and 37\% SO patients died within 12 months. Conclusions Being reviewed in an integrated COPC compared to SO clinic appears to reduce the number and length of hospital admissions for advanced cancer patients. Spending less time in hospitals is important for patients, potentially increasing quality of life. Unsurprisingly, symptoms/complications of cancer were the main reason for admission, relating more often to complications of treatment in the SO group. More patients in the COPC group were discharged home. Reasons behind this were not explored, but may be due to planning discussions in clinics. Findings from this study may aid service development, but a larger cohort is needed to demonstrate statistical significance.

\section{MULTI-PROFESSIONAL WORKING TO SMOOTH THE INTRODUCTIONS OF ENHANCED SUPPORTIVE CARE (ESC)}

Alison Franks, Sharon Hollyoak, Kathryn Oliver, Andrejez Malaczkosk, Dan Acquah, Jade McKelvie. University Hospitals Coventry and Warwickshire NHS Trust

\subsection{6/bmjspcare-2019-ASP.162}

Background The best of palliative medicine and care is underpinned by multi-professional working to optimise symptom management and quality of life once disease specific treatments are no longer appropriate. However, physical and emotional issues are common much earlier in the disease trajectory, during diagnosis and challenging treatments, but aren't always addressed as vigorously as later. The concept of Enhanced Supportive Care is to change this, offering the benefits of good palliative care and medicine alongside active anti-cancer treatments. There's international evidence that this can lead to improved symptom management, quality of life and survival. Thus, NHS England is championing a national pilot, of which we're part, offering ALL patients receiving systemic anti-cancer therapies with palliative intent referral to the ESC team.

Method Discussions with oncologists, palliative care teams, cancer site specific nurses and chemotherapy team, explaining the background and concept. Working closely with oncology IT team designing electronic referral and data collection pathways. Team development - initially a palliative care and oncology trained nurse supported by a palliative oncology consultant, later adding a GP.

'Tools'- King's Fund Patient Outcome Score (IPOS) - primary tool and Hospital Anxiety and Depression Score (HADS).

Changing mind-sets promoting PRO-active rather than REactive referrals.

Results Rising referrals - 35 in February, 72 in November. Reduction of scores (improvements) in all domains of IPOS, other than nausea and fatigue, improved quality of life and avoided admissions.

Conclusion and future Introducing this new service has been challenging and exciting, involving broadening the 'multi-disciplinary team'concept, diplomacy, tact and collaborative working, promoting our skills as an additional benefit, not in competition with other professionals, focusing on the patients quality of life. Our long-term goal is remit expansion to all patients receiving anti-cancer therapies, irrespective of disease stage and treatment intent.

\section{Survivorship and Rehabilitation | Posters $140-142$}

\section{THE EFFECTIVENESS OF CIRCUIT TRAINING ON PHYSICAL ABILITY IN PALLIATIVE CARE PATIENTS}

Aileen McCartney. Medway Community Healthcare CIC

\subsection{6/bmjspcare-2019-ASP.163}

Background Structure exercise groups within palliative care are becoming a more common feature of standard hospice provision, supported by increasing evidence of the benefits for patients on both their physical and emotional function. This small audit reviews the circuit group running at the Wisdom Hospice over a 2 year period, analysing the outcomes for all patients completing the 6 week programme.

Methods All patients joining the circuit group are assessed using 3 physical measures and 1 quality of life measure, prior to starting. These measures are then repeated every 6 weeks while the patient is attending. The number of patients attending the group between 2017 and 2018 were recorded and from those the data of patients who had completed at least 6 weeks and had 6 week outcome measure data were analysed for the audit. There were 28 patients who had attended the group for between 1 and 5 sessions in the same time period.

Outcomes There were 7 patients who completed at least 6 weeks of circuit training between January 2017 and November 2018. All of these patients showed improvements in their physical outcome measures and all showed maintenance or improvement of their quality of life measure. The physical improvements showed an average of $33 \%$ in the Timed Up and Go Test; $68 \%$ in the 2 min timed walk test; and $29 \%$ in the functional reach test. The quality of life measure EORTCQLQ-PAL showed an average improvement of 1 point in both the sections.

A large number of patients who attended for less than 5 sessions all dropped out due to becoming less well.

Conclusions This audit further supports the evidence that palliative care patients can undertake cardiovascular, strength and balance training with significant improvements in physical function. It also highlights the need for early referral into such programmes. 